\title{
Talent and Innovation, Effects of the „Supersonic Management" in the Boeing Company
}

\author{
Gabriela OPAIT ${ }^{\star}$
}

\begin{tabular}{l}
\hline \multicolumn{1}{c}{ A R T I C L E I N F O } \\
\hline Article history: \\
Accepted December 2019 \\
Available online December 2019 \\
\hline JEL Classification \\
C1, C12, C2 \\
Keywords: \\
Logistics, Satellits, Military and \\
commercial aircrafts, Advanced \\
communication systems \\
\hline
\end{tabular}

\begin{abstract}
A B S T R A C T
Boeing is the greatest company from the aerospace field with an expansion in 70 countries. The Boeing Company has three vectors of influence in the "galaxy of the businesses" namely: the first vector with the target in the merchandising of the jetliners; the second vector with the action in defense, space and security systems and the third vector with the "fingerprint" in the supply of services. The diagnostic analysis of the Boeing Company shows that she is a firm with economic performance, because the top management inserts in research, in each year, more than 3 billlions of dollars.
\end{abstract}

(C) 2019 EAI. All rights reserved.

\section{Introduction}

The Boeing Company which is a true „aerospace jewel”, was established in 1916 by William Edward Boeing, in Seattle and meanwhile the seat was moved in Chicago. The Boeing Company is the second provider of the Pentagon, concerning the aerospace and technological equipment. We can see at the Boeing Company, along of the time, a „boom of talent and innovation”. The Boeing Company achieved the 737, 747, 767, 777, 787, 737 Max 9, 787-10 Boeings aircrafts. As brand new, the Boeing Company released the Boeing Analyt X, the Boeing Horizon X and the Boeing T-7A Red Hawk. This statistical approach, focused on the Boeing Company, pursues as aim the making of the prediction which visa the number of Boeing aircrafts in 2019. In the first area of this original study, it observes how we can estimate the number of Boeing aircrafts in 2019. In the second area of this statistical approach, we view the technique whereby we can achieve the architecture of the route which describes the values regarding the Boeing worldwide revenues, in the spell of time 2007-2018. For touch and perform the objective of this statistical processing, we observe as spent method the prognosis through the „Least Squares Method”. Johann Carl Friedrich Gauss accomplished, in 1823, the „Least Squares Method” which represents the „engine” through we can identify the „profiles” of the values which belong to the equations's parameters.

2. The statistical processing which reflects the estimation in 2019 concerning the number of Boenig aircrafts

Table 1. The string concerning the number of Boeing aircrafts, in the spell of time 1998-2018

\begin{tabular}{|c|c|c|c|}
\hline YEARS & $\begin{array}{c}\text { BOEING'S AIRCRAFTS } \\
\left(\xi_{i}\right)\end{array}$ & YEARS & BOEING'S AIRCRAFTS \\
$\left(\xi_{i}\right)$ & 481 \\
\hline 1998 & 564 & 2009 & 462 \\
\hline 1999 & 620 & 2010 & 477 \\
\hline 2000 & 492 & 2011 & 601 \\
\hline 2001 & 527 & 2012 & 648 \\
\hline 2002 & 381 & 2013 & 723 \\
\hline 2003 & 281 & 2014 & 762 \\
\hline 2004 & 285 & 2015 & 748 \\
\hline 2005 & 290 & 2016 & $\mathbf{7 6 3}$ \\
\hline 2006 & 398 & 2017 & 806 \\
\hline 2007 & 441 & 2018 & \\
\hline 2008 & 375 & & \\
\hline
\end{tabular}

Source: „Statista Portal the United States of America” 
- if the technique of the processing for the $\xi$ variable, where $\xi=$ the number of Boeing aircrafts, „stylizes” a linear route $\xi_{t_{i}}=a+b \cdot t_{i}, a$ and $b$ will be [2]:
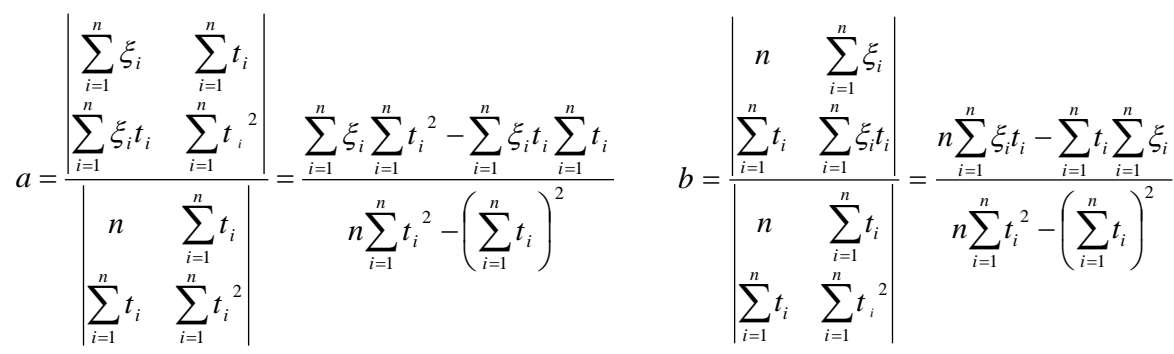

Table 2. The string regarding the number of Boeing aircrafts, if this "galaxy" indicates a linear route

\begin{tabular}{|c|c|c|c|c|c|c|}
\hline YEARS & $\begin{array}{c}\text { BOEING'S } \\
\text { AIRCRAFTS } \\
\left(\xi_{i}\right)\end{array}$ & \multicolumn{5}{|c|}{ LINEAR TENDENCY } \\
\hline 1998 & 564 & -10 & 100 & -5640 & 357,2943723 & 207 \\
\hline 1999 & 620 & -9 & 81 & -5580 & 374,5411256 & 245 \\
\hline 2001 & 527 & -7 & 49 & -3689 & 409,0346321 & 118 \\
\hline 2002 & 381 & -6 & 36 & -2286 & 426,2813853 & 45 \\
\hline 2003 & 281 & -5 & 25 & -1405 & 443,5281386 & 163 \\
\hline 2004 & 285 & -4 & 16 & -1140 & 460,7748918 & 176 \\
\hline 2005 & 290 & -3 & 9 & -870 & 478,0216451 & 188 \\
\hline 2006 & 398 & -2 & 4 & -796 & 495,2683983 & 97 \\
\hline 2010 & 462 & +2 & 4 & 924 & 564,2554113 & 102 \\
\hline 2011 & 477 & +3 & 9 & 1431 & 581,5021646 & 105 \\
\hline 2012 & 601 & +4 & 16 & 2404 & 598,7489178 & 2 \\
\hline 2013 & 648 & +5 & 25 & 3240 & 615,9956711 & 32 \\
\hline 2014 & 723 & +6 & 36 & 4338 & 633,2424243 & 90 \\
\hline 2015 & 762 & +7 & 49 & 5334 & 650,4891776 & 112 \\
\hline 2016 & 748 & +8 & 64 & 5984 & 667,7359308 & 80 \\
\hline 2017 & 763 & +9 & 81 & 6867 & 684,9826841 & 78 \\
\hline 2018 & 806 & +10 & 100 & 8060 & 702,2294373 & 104 \\
\hline TOTAL & 11125 & & 770 & 13280 & 11125 & 2337 \\
\hline
\end{tabular}

$$
\begin{gathered}
a=\frac{11125 \cdot 770-13280 \cdot 0}{21 \cdot 770-0^{2}}=529,7619048 \\
b=\frac{21 \cdot 13280-0 \cdot 11125}{21 \cdot 770-0^{2}}=17,24675325 \\
v_{I}=\left[\frac{\sum_{i=1}^{m}\left|\xi_{i}-\xi_{t_{i}}^{I}\right|}{n}: \frac{\sum_{i=1}^{m} \xi_{i}}{n}\right] \cdot 100=\frac{\sum_{i=1}^{m}\left|\xi_{i}-\xi_{t_{i}}\right|}{\sum_{i=1}^{m} \xi_{i}} \cdot 100=\frac{2337}{11125} \cdot 100=21,01 \%
\end{gathered}
$$

- if the technique of the processing for $\xi$ variable, where $\xi=$ the number of Boeing airfcrafts, „stylizes” a parabolic route $\xi_{t_{i}}=a+b \cdot t_{i}+c t_{i}^{2}, a$ and $b$ will be [2]:

$$
a=\frac{\sum_{i=1}^{n} t_{i}^{4} \sum_{i=1}^{n} \xi_{i}-\sum_{i=1}^{n} t_{i}^{2} \sum_{i=1}^{n} t_{i}^{2} \cdot \xi_{i}}{n \sum_{i=1}^{n} t_{i}{ }^{4}-\left(\sum_{i=1}^{n} t_{i}^{2}\right)^{2}} ; \quad b=\frac{\sum_{i=1}^{n} \xi_{i} t_{i}}{\sum_{i=1}^{n} t_{i}{ }^{2}} ; c=\frac{n \cdot \sum_{i=1}^{n} t_{i}^{2} \cdot \xi_{i}-\sum_{i=1}^{n} t_{i}^{2} \cdot \sum_{i=1}^{n} \xi_{i}}{n \sum_{i=1}^{n} t_{i}{ }^{4}-\left(\sum_{i=1}^{n} t_{i}\right)^{2}}
$$


Table 3. The string concerning the number of Boeing aircrafts, if this „galaxy" indicates a quadratic route

\begin{tabular}{|c|c|c|c|c|c|c|c|c|}
\hline \multirow{2}{*}{ YEARS } & \multirow{2}{*}{$\begin{array}{c}\text { BOEING'S } \\
\text { AIRCRAFTS } \\
\left(\xi_{i}\right)\end{array}$} & \multicolumn{7}{|c|}{ PARABOLIC TENDENCY } \\
\hline & & $t_{i}$ & $t_{i}^{2}$ & $t_{i}^{3}$ & $t_{i}^{4}$ & $t_{i}^{2} \xi_{i}$ & $\xi_{t_{i}}=a+b t_{i}+c t_{i}^{2}$ & $\left|\xi_{i}-\xi_{t_{i}}\right|$ \\
\hline 1998 & 564 & -10 & 100 & -1000 & 10000 & 56400 & 560,6747600 & 3 \\
\hline 1999 & 620 & -9 & 81 & -729 & 6561 & 50220 & 516,9073969 & 103 \\
\hline 2000 & 492 & -8 & 64 & -512 & 4096 & 31488 & 479,5625724 & 12 \\
\hline 2001 & 527 & -7 & 49 & -343 & 2401 & 25823 & 448,6402865 & 78 \\
\hline 2002 & 381 & -6 & 36 & -216 & 1296 & 13716 & 424,1405391 & 43 \\
\hline 2003 & 281 & -5 & 25 & -125 & 625 & 7025 & 406,0633303 & 125 \\
\hline 2004 & 285 & -4 & 16 & -64 & 256 & 4560 & 394,4086600 & 109 \\
\hline 2005 & 290 & -3 & 9 & -27 & 81 & 2610 & 389,1765283 & 99 \\
\hline 2006 & 398 & -2 & 4 & -8 & 16 & 1592 & 390,3669351 & 8 \\
\hline 2007 & 441 & -1 & 1 & -1 & 1 & 441 & 397,9798805 & 43 \\
\hline 2008 & 375 & 0 & 0 & 0 & 0 & 0 & 412,0153645 & 37 \\
\hline 2009 & 481 & +1 & 1 & 1 & 1 & 481 & 432,4733870 & 49 \\
\hline 2010 & 462 & +2 & 4 & 8 & 16 & 1848 & 459,3539481 & 3 \\
\hline 2011 & 477 & +3 & 9 & 27 & 81 & 4293 & 515,1359327 & 38 \\
\hline 2012 & 601 & +4 & 16 & 64 & 256 & 9616 & 532,3826860 & 69 \\
\hline 2013 & 648 & +5 & 25 & 125 & 625 & 16200 & 578,5308628 & 69 \\
\hline 2014 & 723 & +6 & 36 & 216 & 1296 & 26028 & 631,1015781 & 92 \\
\hline 2015 & 762 & +7 & 49 & 343 & 2401 & 37338 & 690,0948320 & 72 \\
\hline 2016 & 748 & +8 & 64 & 512 & 4096 & 47872 & 755,5106244 & 8 \\
\hline 2017 & 763 & +9 & 81 & 729 & 6561 & 61803 & 827,3489554 & 64 \\
\hline 2018 & 806 & +10 & 100 & 1000 & 10000 & 80600 & 905,6098250 & 100 \\
\hline TOTAL & 11125 & & 770 & & 50666 & 479954 & & 1224 \\
\hline
\end{tabular}

$$
\begin{gathered}
a=\frac{\sum_{i=1}^{n} t_{i}^{4} \sum_{i=1}^{n} \xi_{i}-\sum_{i=1}^{n} t_{i}^{2} \sum_{i=1}^{n} t_{i}^{2} \cdot \xi_{i}}{n \sum_{i=1}^{n} t_{i}^{4}-\left(\sum_{i=1}^{n} t_{i}^{2}\right)^{2}}=\frac{50666 \cdot 11125-770 \cdot 479954}{21 \cdot 50666-770^{2}}=412,0153645 \\
b=\frac{\sum_{i=1}^{n} \xi_{i} t_{i}}{\sum_{i=1}^{n} t_{i}^{2}}=\frac{13280}{770}=17,24675325 \\
c=\frac{n \cdot \sum_{i=1}^{n} t_{i}^{2} \cdot \xi_{i}-\sum_{i=1}^{n} t_{i}^{2} \cdot \sum_{i=1}^{n} \xi_{i}}{n \sum_{i=1}^{n} t_{i}{ }^{4}-\left(\sum_{i=1}^{n} t_{i}^{2}\right)^{2}}=\frac{21 \cdot 479954-770 \cdot 11125}{21 \cdot 50666-770^{2}}=3,21126928 \\
v_{I I}=\left[\frac{\sum_{i=1}^{m}\left|\xi_{i}-\xi_{t_{i}}\right|}{n}: \frac{\sum_{i=1}^{m} \xi_{i}}{n}\right] \cdot 100=\frac{\sum_{i=1}^{m}\left|\xi_{i}-\xi_{t_{i}}\right|}{\sum_{i=1}^{m} \xi_{i}} \cdot 100=\frac{1224}{11125} \cdot 100=11 \%
\end{gathered}
$$

- if the technique of the processing for $\xi$ variable, where $\xi=$ the number of Boeing aircrafts, „stylizes” a parabolic route of three degree $\xi_{t_{i}}=a+b \cdot t_{i}+c t_{i}^{2}+d t_{i}^{3}, a, b, c$ and $d$ will be [2]: 
Table 4. The string regarding the number of Boeing aircrafts,

if this "galaxy" indicates a quadratic route of three degree

\begin{tabular}{|c|c|c|c|c|c|c|c|c|c|}
\hline \multirow{2}{*}{ YEARS } & \multirow{2}{*}{$\begin{array}{c}\text { BOEING'S } \\
\text { AIRCRAFTS } \\
\left(\xi_{i}\right)\end{array}$} & \multicolumn{8}{|c|}{ PARABOLIC TENDENCY OF THREE DEGREE } \\
\hline & & $t_{i}$ & $t_{i}^{2}$ & $t_{i}^{3}$ & $t_{i}^{4}$ & $t_{i}^{6}$ & $t_{i}^{3} \xi_{i}$ & $\xi_{t_{i}}=a+b \cdot t_{i}+c t_{i}^{2}+d t_{i}^{3}$ & $\left|\xi_{i}-\xi_{t_{i}}\right|$ \\
\hline 1998 & 564 & -10 & 100 & -1000 & 10000 & 1000000 & -564000 & 651,1793711 & 87 \\
\hline 1999 & 620 & -9 & 81 & -729 & 6561 & 531441 & -451980 & 554,3092413 & 66 \\
\hline 2000 & 492 & -8 & 64 & -512 & 4096 & 262144 & -251904 & 475,6255361 & 16 \\
\hline 2001 & 527 & -7 & 49 & -343 & 2401 & 117649 & -180761 & 416,4878236 & 111 \\
\hline 2002 & 381 & -6 & 36 & -216 & 1296 & 46656 & -82296 & 375,2556721 & 54 \\
\hline 2003 & 281 & -5 & 25 & -125 & 625 & 15625 & -35125 & 350,2886498 & 69 \\
\hline 2004 & 285 & -4 & 16 & -64 & 256 & 4096 & -18240 & 339,9463250 & 55 \\
\hline 2005 & 290 & -3 & 9 & -27 & 81 & 729 & -7830 & 342,5882658 & 53 \\
\hline 2006 & 398 & -2 & 4 & -8 & 16 & 64 & -3184 & 356,5740405 & 41 \\
\hline 2007 & 441 & -1 & 1 & -1 & 1 & 1 & -441 & 380,2632173 & 61 \\
\hline 2008 & 375 & 0 & 0 & 0 & 0 & 0 & 0 & 412,0153645 & 37 \\
\hline 2009 & 481 & +1 & 1 & 1 & 1 & 1 & 481 & 450,1900502 & 31 \\
\hline 2010 & 462 & +2 & 4 & 8 & 16 & 64 & 3696 & 493,1468427 & 31 \\
\hline 2011 & 477 & +3 & 9 & 27 & 81 & 729 & 12879 & 539,2453102 & 62 \\
\hline 2012 & 601 & +4 & 16 & 64 & 256 & 4096 & 38464 & 586,8450210 & 14 \\
\hline 2013 & 648 & +5 & 25 & 125 & 625 & 15625 & 81000 & 634,3055432 & 14 \\
\hline 2014 & 723 & +6 & 36 & 216 & 1296 & 46656 & 156168 & 679,9864451 & 43 \\
\hline 2015 & 762 & +7 & 49 & 343 & 2401 & 117649 & 261366 & 722,2472949 & 40 \\
\hline 2016 & 748 & +8 & 64 & 512 & 4096 & 262144 & 382976 & 759,4476608 & 11 \\
\hline 2017 & 763 & +9 & 81 & 729 & 6561 & 531441 & 556227 & 789,9471111 & 27 \\
\hline 2018 & 806 & +10 & 100 & 1000 & 10000 & 1000000 & 806000 & 812,1052139 & 6 \\
\hline TOTAL & 11125 & & 770 & & 50666 & 3956810 & 703496 & & 929 \\
\hline
\end{tabular}

$$
\begin{aligned}
& a=\frac{\sum_{i=1}^{n} t_{i}^{4} \sum_{i=1}^{n} \xi_{i}-\sum_{i=1}^{n} t_{i}^{2} \sum_{i=1}^{n} t_{i}^{2} \cdot \xi_{i}}{n \sum_{i=1}^{n} t_{i}^{4}-\left(\sum_{i=1}^{n} t_{i}^{2}\right)^{2}}=\frac{50666 \cdot 11125-770 \cdot 479954}{21 \cdot 50666-770^{2}}=412,0153645 \\
& b=\frac{\sum_{i=1}^{n} t_{i}^{6} \cdot \sum_{i=1}^{n} t_{i} \cdot \xi_{i}-\sum_{i=1}^{n} t_{i}^{4} \cdot \sum_{i=1}^{n} t_{i}^{3} \cdot \xi_{i}}{\sum_{i=1}^{n} t_{i}^{2} \cdot \sum_{i=1}^{n} t_{i}^{6}-\left(\sum_{i=1}^{n} t_{i}^{4}\right)^{2}}=\frac{3956810 \cdot 13280-50666 \cdot 703496}{770 \cdot 3956810-50666^{2}}=35,23682174 \\
& c=\frac{n \cdot \sum_{i=1}^{n} t_{i}^{2} \cdot \xi_{i}-\sum_{i=1}^{n} t_{i}^{2} \cdot \sum_{i=1}^{n} \xi_{i}}{n \sum_{i=1}^{n} t_{i}{ }^{4}-\left(\sum_{i=1}^{n} t_{i}^{2}\right)^{2}}=\frac{21 \cdot 479954-770 \cdot 11125}{21 \cdot 50666-770^{2}}=3,21126928 \\
& d=\frac{\sum_{i=1}^{n} t_{i}^{2} \cdot \sum_{i=1}^{n} t_{i}^{3} \cdot \xi_{i}-\sum_{i=1}^{n} t_{i}^{4} \cdot \sum_{i=1}^{n} t_{i} \cdot \xi_{i}}{\sum_{i=1}^{n} t_{i}^{2} \cdot \sum_{i=1}^{n} t_{i}^{6}-\left(\sum_{i=1}^{n} t_{i}^{4}\right)^{2}}=\frac{770 \cdot 703496-50666 \cdot 13280}{770 \cdot 3956810-50666^{2}}=-0,273405296 \\
& v_{I I I}=\left[\frac{\sum_{i=1}^{m}\left|\xi_{i}-\xi_{t_{i}}^{I I I}\right|}{n}: \frac{\sum_{i=1}^{m} \xi_{i}}{n}\right] \cdot 100=\frac{\sum_{i=1}^{m}\left|\xi_{i}-\xi_{t_{i}}^{I I I}\right|}{\sum_{i=1}^{m} \xi_{i}} \cdot 100=\frac{929}{11125} \cdot 100=8,35 \%
\end{aligned}
$$

- if the technique of the processing for $\xi$ variable, where $\xi=$ the number of Boeing aircrafts, „stylizes” an exponential route $\xi_{t_{i}}=a b^{t_{i}}, a$ and $b$ will be [2]: 


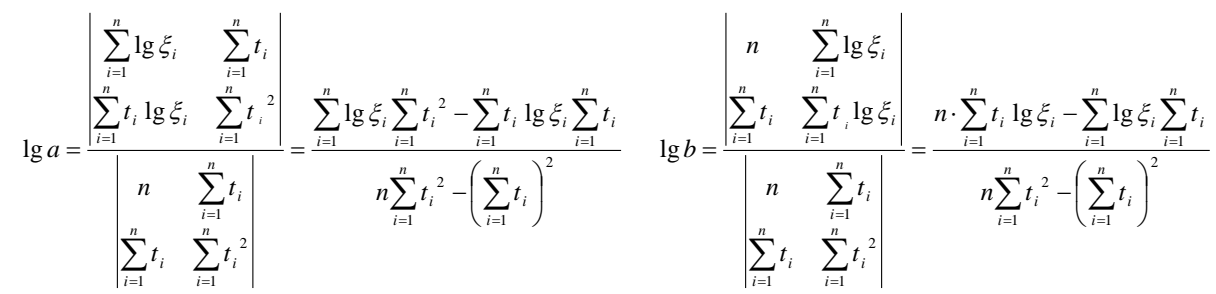

Table 5. The string concerning the number of Boeing aircrafts, if this „galaxy” indicates an exponential route

\begin{tabular}{|c|c|c|c|c|c|c|}
\hline \multirow{2}{*}{ YEARS } & \multirow{2}{*}{$\begin{array}{c}\text { BOEING'S } \\
\text { AIRCRAFTS } \\
\left(\xi_{i}\right)\end{array}$} & \multicolumn{5}{|c|}{ EXPONENTIAL TENDENCY } \\
\hline & & $\lg \xi_{i}$ & $t_{i} \lg \xi_{i}$ & $\lg \xi_{t_{i}}=\lg a+t_{i} \lg b$ & $\xi_{t_{i}}=a b^{t_{i}}$ & $\left|\xi_{i}-\xi_{t_{i}}\right|$ \\
\hline 1998 & 564 & 2,751279104 & $-27,51279104$ & 2,565213674 & 367,4630488 & 197 \\
\hline 1999 & 620 & 2,792391690 & $-25,13152521$ & 2,578893654 & 379,2221132 & 241 \\
\hline 2000 & 492 & 2,691965103 & $-21,53572082$ & 2,592573634 & 391,3574756 & 101 \\
\hline 2001 & 527 & 2,721810615 & $-19,05267431$ & 2,606253614 & 403,8811777 & 123 \\
\hline 2002 & 381 & 2,580924976 & $-15,48554985$ & 2,619933594 & 416,8056467 & 36 \\
\hline 2003 & 281 & 2,448706320 & $-12,24353160$ & 2,633613574 & 430,1437074 & 149 \\
\hline 2004 & 285 & 2,454844860 & $-9,819379440$ & 2,647293554 & 443,9085950 & 159 \\
\hline 2005 & 290 & 2,462397998 & $-7,387193994$ & 2,660973534 & 458,1139683 & 168 \\
\hline 2006 & 398 & 2,599883072 & $-5,199766144$ & 2,674653514 & 472,7739230 & 75 \\
\hline 2007 & 441 & 2,644438589 & $-2,644438589$ & 2,688333494 & 487,9030062 & 47 \\
\hline 2008 & 375 & 2,574031268 & 0 & 2,702013474 & 503,5162302 & 129 \\
\hline 2009 & 481 & 2,682145076 & 2,682145076 & 2,715693454 & 519,6290878 & 39 \\
\hline 2010 & 462 & 2,664641976 & 5,329283951 & 2,729373434 & 536,2575677 & 74 \\
\hline 2011 & 477 & 2,678518379 & 8,035555137 & 2,743053414 & 553,4181701 & 76 \\
\hline 2012 & 601 & 2,778874472 & 11,11549789 & 2,756733394 & 571,1279233 & 30 \\
\hline 2013 & 648 & 2,811575006 & 14,05787503 & 2,770413374 & 589,4044005 & 59 \\
\hline 2014 & 723 & 2,859138297 & 17,15482978 & 2,784093354 & 608,2657373 & 115 \\
\hline 2015 & 762 & 2,881954971 & 20,17368480 & 2,797773334 & 627,7306495 & 134 \\
\hline 2016 & 748 & 2,873901598 & 22,99121278 & 2,811453314 & 647,8184520 & 100 \\
\hline 2017 & 763 & 2,882524538 & 25,94272084 & 2,825133294 & 668,5490777 & 94 \\
\hline 2018 & 806 & 2,906335042 & 29,06335042 & 2,838813274 & 689,9430973 & 116 \\
\hline TOTAL & 11125 & 56,74228295 & 10,53358471 & & & 2262 \\
\hline
\end{tabular}

$$
\begin{gathered}
\lg a=\frac{56,74228295 \cdot 770-10,53358471 \cdot 0}{21 \cdot 770-0^{2}}=2,702013474 \\
\lg b=\frac{21 \cdot 10,53358471-56,74228295 \cdot 0}{21 \cdot 770-0^{2}}=0,01367998 \\
v_{\text {exp }}=\left[\frac{\sum_{i=1}^{n}\left|\xi_{i}-\xi_{t_{i}}^{\exp }\right|}{n}: \frac{\sum_{i=1}^{n} \xi_{i}}{n}\right] \cdot 100=\frac{\sum_{i=1}^{n}\left|\xi_{i}-\xi_{i_{i}}^{\exp }\right|}{\sum_{i=1}^{n} \xi_{i}} \cdot 100=\frac{2262}{11125} \cdot 100=20,33 \% \\
v_{I I I}=8,35 \%<v_{I I}=11 \%<v_{\text {exp }}=20,33 \%<v_{I}=21,01 \%
\end{gathered}
$$

The "mix" of the processing, which has as target the number of Boeing aircrafts, pursues a quadratic route of three degree $\xi_{t_{i}}=a+b \cdot t_{i}+c t_{i}^{2}+d t_{i}^{3}$ 


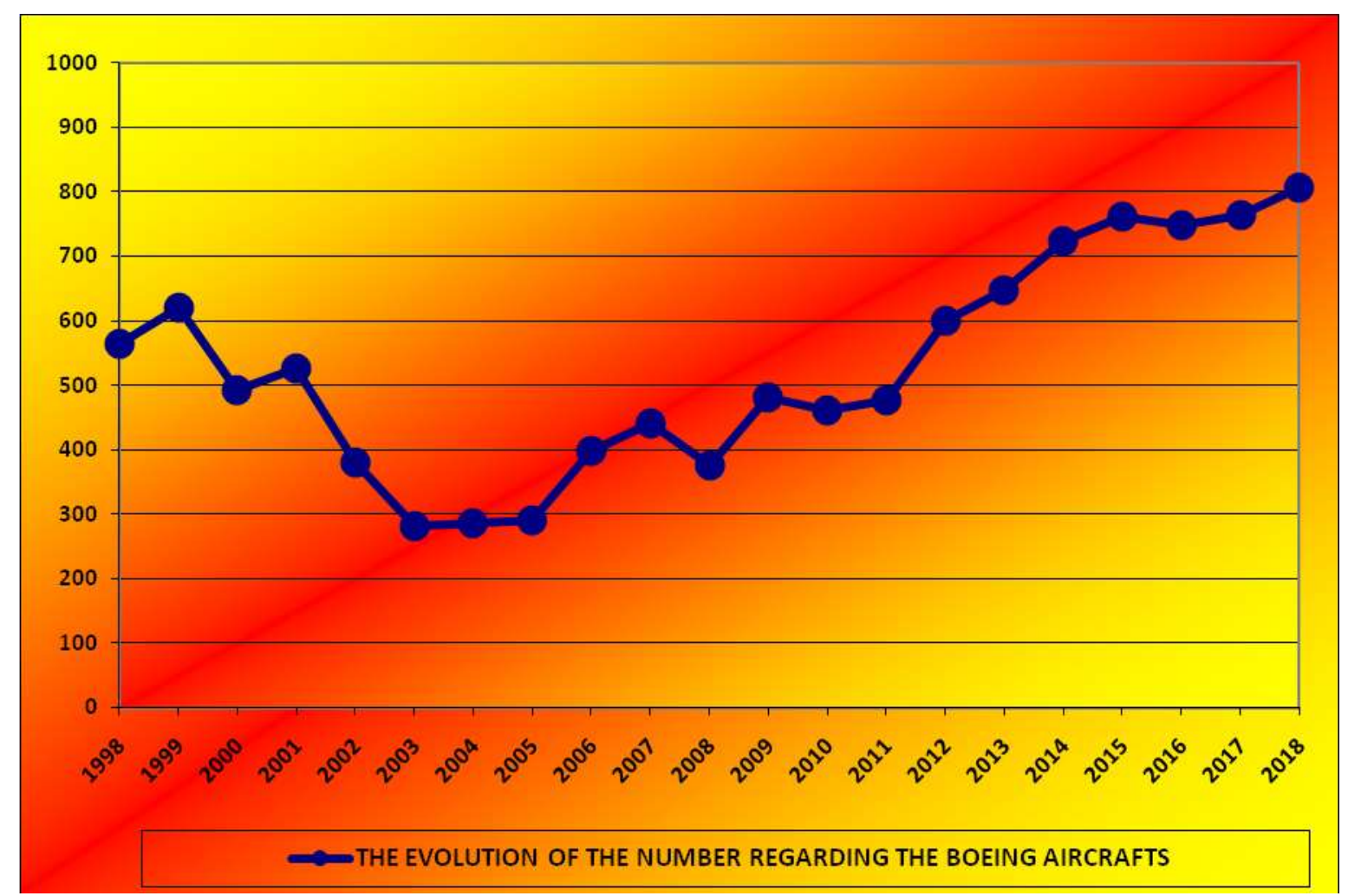

Graph 1. The quadratic route of three degree for the packet unveiled by the values which indicate the dynamic of the number concerning the Boeing aircrafts

$\xi_{2019}^{\text {BOEING_AIRCRAFTS }}=412,0153645+35,23682174 \cdot 11+3,21126928 \cdot 11^{2}+(-0,273405296) \cdot 11^{3}=824,28 \cong 824$

a) 3. The statistical processing which analyses the worldwide revenues of the Boeing Company, in the spell of the time 2007-2018, for to identify them trend

Table 6. The string of numbers regarding the worlwide revenues of the

Boeing Company, in the spell of time 2007-2018

\begin{tabular}{|c|c|}
\hline YEARS & $\begin{array}{c}\text { BOEING'S WORLDWIDE REVENUES } \\
\text { (billions } \$ \\
\left(\omega_{i}\right)\end{array}$ \\
\hline 2007 & $\mathbf{6 6 , 3 8 7}$ \\
\hline 2008 & $\mathbf{6 0 , 9 0 9}$ \\
\hline 2009 & $\mathbf{6 8 , 2 8 1}$ \\
\hline 2010 & $\mathbf{6 4 , 3 0 6}$ \\
\hline 2011 & $\mathbf{6 8 , 7 3 5}$ \\
\hline 2012 & $\mathbf{8 1 , 6 9 8}$ \\
\hline 2013 & $\mathbf{8 6 , 6 2 3}$ \\
\hline 2014 & $\mathbf{9 0 , 7 6 2}$ \\
\hline 2015 & $\mathbf{9 6 , 1 1 4}$ \\
\hline 2016 & $\mathbf{9 3 , 4 9 6}$ \\
\hline 2018 & $\mathbf{9 4 , 0 0 5}$ \\
\hline & Source: „Statista Portal the United States of America” \\
\hline
\end{tabular}

- if the technique of the processing for $\omega$ variable, where $\omega=$ Boeing's worlwide revenues, "stylizes” a linear route $\omega_{t_{i}}=a+b \cdot t_{i}, a$ and $b$ will be [2]: 
Table 7. The string of numbers concerning the Boeing's worldwide revenues, if this serial indicates a linear route

\begin{tabular}{|c|c|c|c|c|c|c|}
\hline \multirow{2}{*}{ YEARS } & \multirow{2}{*}{$\begin{array}{c}\text { BOEING'S WORLDWIDE } \\
\text { REVENUES } \\
\text { (billions \$) } \\
\left(\omega_{i}\right)\end{array}$} & \multicolumn{5}{|c|}{ LINEAR TENDENCY } \\
\hline & & $t_{i}$ & $t_{i}^{2}$ & $t_{i} \omega_{i}$ & $\omega_{t_{i}}=a+b t_{i}$ & $\left|\omega_{i}-\omega_{t_{i}}\right|$ \\
\hline 2007 & 66,387 & -6 & 36 & $-398,322$ & 60,62429029 & 5,763 \\
\hline 2008 & 60,909 & -5 & 25 & $-304,545$ & 64,02639469 & 3,117 \\
\hline 2009 & 68,281 & -4 & 16 & $-273,124$ & 67,42849909 & 0,853 \\
\hline 2010 & 64,306 & -3 & 9 & $-192,918$ & 70,83060348 & 6,524 \\
\hline 2011 & 68,735 & -2 & 4 & $-137,470$ & 74,23270788 & 5,498 \\
\hline 2012 & 81,698 & -1 & 1 & $-81,698$ & 77,63481227 & 4,063 \\
\hline 2013 & 86,623 & +1 & 1 & 86,623 & 84,43902107 & 2,184 \\
\hline 2014 & 90,762 & +2 & 4 & 181,524 & 87,84112546 & 2,921 \\
\hline 2015 & 96,114 & +3 & 9 & 288,342 & 91,24322986 & 4,871 \\
\hline 2016 & 93,496 & +4 & 16 & 373,984 & 94,64533425 & 1,149 \\
\hline 2017 & 94,005 & +5 & 25 & 470,025 & 98,04743865 & 4,042 \\
\hline 2018 & 101,127 & +6 & 36 & 606,762 & 101,4495430 & 0,323 \\
\hline TOTAL & 972,443 & & 182 & 619,183 & 972,443 & 41,308 \\
\hline
\end{tabular}

$$
\begin{gathered}
a=\frac{\sum_{i=1}^{n} \omega_{i} \sum_{i=1}^{n} t_{i}^{2}-\sum_{i=1}^{n} \omega_{i} t_{i} \sum_{i=1}^{n} t_{i}}{n \sum_{i=1}^{n} t_{i}^{2}-\left(\sum_{i=1}^{n} t_{i}\right)^{2}}=\frac{972,443 \cdot 182-619,183 \cdot 0}{12 \cdot 182-0^{2}}=81,03691667 \\
b=\frac{n \sum_{i=1}^{n} \omega_{i} t_{i}-\sum_{i=1}^{n} t_{i} \sum_{i=1}^{n} \omega_{i}}{n \sum_{i=1}^{n} t_{i}^{2}-\left(\sum_{i=1}^{n} t_{i}\right)^{2}}=\frac{12 \cdot 619,183-0 \cdot 972,443}{12 \cdot 182-0^{2}}=3,402104396 \\
v_{I}=\left[\frac{\sum_{i=1}^{n}\left|\omega_{i}-y_{t_{i}}^{I}\right|}{n}: \frac{\sum_{i=1}^{n} \omega_{i}}{n}\right] \cdot 100=\frac{\sum_{i=1}^{n}\left|\omega_{i}-\omega_{t_{i}}^{I}\right|}{\sum_{i=1}^{n} \omega_{i}} \cdot 100=\frac{41,308}{972,443} \cdot 100=4,25 \%
\end{gathered}
$$

\begin{tabular}{|c|c|c|c|c|c|c|c|c|}
\hline \multirow{2}{*}{ YEARS } & \multirow{2}{*}{$\begin{array}{c}\text { BOEING'S } \\
\text { WORLDWIDE } \\
\text { REVENUES } \\
\text { (billions \$) } \\
\left(\omega_{i}\right)\end{array}$} & \multicolumn{7}{|c|}{ PARABOLIC TENDENCY } \\
\hline & & $t_{i}$ & $t_{i}^{2}$ & $t_{i}^{3}$ & $t_{i}^{4}$ & $t_{i}^{2} \omega_{i}$ & $\omega_{t_{i}}=a+b t_{i}+c t_{i}^{2}$ & $\omega_{i}-\omega_{t_{i}}$ \\
\hline 2007 & 66,387 & -6 & 36 & -216 & 1296 & 2389,932 & 60,54462192 & 5,842 \\
\hline 2008 & 60,909 & -5 & 25 & -125 & 625 & 1522,725 & 63,98879122 & 3,080 \\
\hline 2009 & 68,281 & -4 & 16 & -64 & 256 & 1092,496 & 67,42531235 & 0,856 \\
\hline 2010 & 64,306 & -3 & 9 & -27 & 81 & 578,754 & 70,85418532 & 6,548 \\
\hline 2011 & 68,735 & -2 & 4 & -8 & 16 & 274,940 & 74,27541013 & 5,540 \\
\hline 2012 & 81,698 & -1 & 1 & -1 & 1 & 81,698 & 77,68898677 & 4,009 \\
\hline 2013 & 86,623 & +1 & 1 & +1 & 1 & 86,623 & 84,49319556 & 2,130 \\
\hline 2014 & 90,762 & +2 & 4 & +8 & 16 & 363,048 & 87,88382771 & 2,878 \\
\hline 2015 & 96,114 & +3 & 9 & +27 & 81 & 865,026 & 91,26681170 & 4.847 \\
\hline 2016 & 93,496 & +4 & 16 & +64 & 256 & 1495,936 & 94,64214752 & 1,146 \\
\hline 2017 & 94,005 & +5 & 25 & +125 & 625 & 2350,125 & 98,00983518 & 4,005 \\
\hline 2018 & 101,127 & +6 & 36 & +216 & 1296 & 3640,572 & 101,3698747 & 0,243 \\
\hline TOTAL & 972,443 & & 182 & & 4550 & 14741,875 & 972,443 & 41,124 \\
\hline
\end{tabular}

- if the technique of the processing for $\omega$ variable, where $\omega=$ Boeing's worlwide revenues, „stylizes” a quadratic route $\omega_{t_{i}}=a+b \cdot t_{i}+c t_{i}^{2}, a$ and $b$ will be [2]:

Table 8. The string of numbers regarding the Boeing's worldwide revenues, if this serial indicates a quadratic route 


$$
\begin{gathered}
a=\frac{\sum_{i=1}^{n} t_{i}^{4} \sum_{i=1}^{n} \omega_{i}-\sum_{i=1}^{n} t_{i}^{2} \sum_{i=1}^{n} t_{i}^{2} \cdot \omega_{i}}{n \sum_{i=1}^{n} t_{i}^{4}-\left(\sum_{i=1}^{n} t_{i}^{2}\right)^{2}}=\frac{4550 \cdot 972,443-182 \cdot 14741,875}{12 \cdot 4550-182^{2}}=81,09491525 \\
b=\frac{\sum_{i=1}^{n} \omega_{i} t_{i}}{\sum_{i=1}^{n} t_{i}^{2}}=\frac{619,183}{182}=3,402104396 \\
c=\frac{n \cdot \sum_{i=1}^{n} t_{i}^{2} \cdot \omega_{i}-\sum_{i=1}^{n} t_{i}^{2} \cdot \sum_{i=1}^{n} \omega_{i}}{n \sum_{i=1}^{n} t_{i}^{4}-\left(\sum_{i=1}^{n} t_{i}^{2}\right)^{2}}=\frac{12 \cdot 14741,875-182 \cdot 972,443}{12 \cdot 4550-182^{2}}=-0,003824082 \\
v_{I I}=\left[\frac{\sum_{i=1}^{n}\left|\omega_{i}-\omega_{t_{i}}^{I I}\right|}{n}: \frac{\sum_{i=1}^{n} \omega_{i}}{n}\right] \cdot 100=\frac{\sum_{i=1}^{n}\left|\omega_{i}-\omega_{t_{i}}^{I I}\right|}{\sum_{i=1}^{n} \omega_{i}} \cdot 100=\frac{41,124}{972,443} \cdot 100=4,23 \%
\end{gathered}
$$

- if the technique of the processing for $\omega$ variable, where $\omega=$ Boeing's worlwide revenues, „stylizes” a

\begin{tabular}{|c|c|c|c|c|c|c|c|c|c|}
\hline \multirow{2}{*}{ YEARS } & \multirow{2}{*}{$\begin{array}{c}\text { BOEING'S } \\
\text { WORLDWIDE } \\
\text { REVENUES } \\
\text { (billions \$) } \\
\left(\omega_{i}\right)\end{array}$} & \multicolumn{8}{|c|}{ PARABOLIC TENDENCY OF THREE DEGREE } \\
\hline & & $t_{i}$ & $t_{i}^{2}$ & $t_{i}^{3}$ & $t_{i}^{4}$ & $t_{i}^{6}$ & $t_{i}^{3} \omega_{i}$ & $\omega_{t_{i}}=a+b \cdot t_{i}+c t_{i}^{2}+d t_{i}^{3}$ & $\left|\omega_{i}-\omega_{t_{i}}\right|$ \\
\hline 2007 & 66,387 & -6 & 36 & -216 & 1296 & 46656 & $-14339,592$ & 64,34275650 & 2,044 \\
\hline 2008 & 60,909 & -5 & 25 & -125 & 625 & 15625 & $-7613,625$ & 63,98879120 & 3.080 \\
\hline 2009 & 68,281 & -4 & 16 & -64 & 256 & 4096 & $-4369,984$ & 65,35360255 & 2,927 \\
\hline 2010 & 64,306 & -3 & 9 & -27 & 81 & 729 & $-1736,262$ & 68,09190560 & 3,786 \\
\hline 2011 & 68,735 & -2 & 4 & -8 & 16 & 64 & $-549,880$ & 71,85841537 & 3,123 \\
\hline 2012 & 81,698 & -1 & 1 & -1 & 1 & 1 & $-81,698$ & 76,30784691 & 5,390 \\
\hline 2013 & 86,623 & +1 & 1 & +1 & 1 & 1 & 86,623 & 85,87433543 & 0,749 \\
\hline 2014 & 90,762 & +2 & 4 & +8 & 16 & 64 & 726,096 & 90,30082247 & 0,461 \\
\hline 2015 & 96,114 & +3 & 9 & +27 & 81 & 729 & 2595,078 & 94,02909143 & 2,085 \\
\hline 2016 & 93,496 & +4 & 16 & +64 & 256 & 4096 & 5983,744 & 96,71385733 & 3,218 \\
\hline 2017 & 94,005 & +5 & 25 & +125 & 625 & 15625 & 11750,625 & 98,00983521 & 4,005 \\
\hline 2018 & 101,127 & +6 & 36 & +216 & 1296 & 46656 & 21843,432 & 97,57174010 & 3,555 \\
\hline TOTAL & 972,443 & & 182 & & 4550 & 134342 & 14294,557 & 972,443 & 34,423 \\
\hline
\end{tabular}
parabolic route of three degree $\omega_{t_{i}}=a+b \cdot t_{i}+c t_{i}^{2}+d t_{i}^{3}, a, b, c$ and $d$ will be [2]:

Table 9. The string of numbers concerning the Boeing's worldwide revenues, if this serial indicates a quadratic route of three degree

$$
\begin{aligned}
& a=\frac{\sum_{i=1}^{n} t_{i}^{4} \sum_{i=1}^{n} \omega_{i}-\sum_{i=1}^{n} t_{i}^{2} \sum_{i=1}^{n} t_{i}^{2} \cdot \omega_{i}}{n \sum_{i=1}^{n} t_{i}{ }^{4}-\left(\sum_{i=1}^{n} t_{i}^{2}\right)^{2}}=\frac{4550 \cdot 972,443-182 \cdot 14741,875}{12 \cdot 4550-182^{2}}=81,09491525 \\
& b=\frac{\sum_{i=1}^{n} t_{i}^{6} \cdot \sum_{i=1}^{n} t_{i} \cdot \omega_{i}-\sum_{i=1}^{n} t_{i}^{4} \cdot \sum_{i=1}^{n} t_{i}^{3} \cdot \omega_{i}}{\sum_{i=1}^{n} t_{i}{ }^{2} \cdot \sum_{i=1}^{n} t_{i}^{6}-\left(\sum_{i=1}^{n} t_{i}^{4}\right)^{2}}=\frac{134342 \cdot 619,183-4550 \cdot 14294,557}{182 \cdot 134342-4550^{2}}=4,840791751
\end{aligned}
$$




$$
\begin{gathered}
c=\frac{n \cdot \sum_{i=1}^{n} t_{i}^{2} \cdot \omega_{i}-\sum_{i=1}^{n} t_{i}^{2} \cdot \sum_{i=1}^{n} \omega_{i}}{n \sum_{i=1}^{n} t_{i}^{4}-\left(\sum_{i=1}^{n} t_{i}^{2}\right)^{2}}=\frac{12 \cdot 14741,875-182 \cdot 972,443}{12 \cdot 4550-182^{2}}=-0,003824082 \\
d=\frac{\sum_{i=1}^{n} t_{i}^{2} \cdot \sum_{i=1}^{n} t_{i}^{3} \cdot \omega_{i}-\sum_{i=1}^{n} t_{i}^{4} \cdot \sum_{i=1}^{n} t_{i} \cdot \omega_{i}}{\sum_{i=1}^{n} t_{i}^{2} \cdot \sum_{i=1}^{n} t_{i}^{6}-\left(\sum_{i=1}^{n} t_{i}^{4}\right)^{2}}=\frac{182 \cdot 14294,557-4550 \cdot 619,183}{182 \cdot 134342-4550^{2}}=-0,057547494 \\
v_{I I I}=\left[\frac{\sum_{i=1}^{m}\left|\omega_{i}-\omega_{t_{i}}^{I I I}\right|}{n}: \frac{\left.\sum_{i=1}^{m} \omega_{i}\right]}{n}\right] \cdot 100=\frac{\sum_{i=1}^{m}\left|\omega_{i}-\omega_{t_{i}}^{I I I}\right|}{\sum_{i=1}^{m} \omega_{i}} \cdot 100=\frac{34,423}{972,443} \cdot 100=3,54 \%
\end{gathered}
$$

- if the technique of the processing for $\omega$ variable, where $\omega=$ Boeing's worlwide revenues, "stylizes" an

\begin{tabular}{|c|c|c|c|c|c|c|}
\hline \multirow{2}{*}{ YEARS } & \multirow{2}{*}{$\begin{array}{c}\text { BOEING'S } \\
\text { WORLDWIDE } \\
\text { REVENUES } \\
\text { (billions \$) } \\
\left(\omega_{i}\right)\end{array}$} & \multicolumn{5}{|c|}{ EXPONENTIAL TENDENCY } \\
\hline & & $\lg \omega_{i}$ & $t_{i} \lg \omega_{i}$ & $\lg \omega_{t_{i}}=\lg a+t_{i} \lg b$ & $\omega_{t_{i}}=a b^{t_{i}}$ & $\omega_{i}-\omega_{t_{i}}$ \\
\hline 2007 & 66,387 & 1,822083044 & $-10,93249826$ & 1,790873007 & 61,78357110 & 4,603 \\
\hline 2008 & 60,909 & 1,784681469 & $-8,923407347$ & 1,809419732 & 64,47921359 & 3,570 \\
\hline 2009 & 68,281 & 1,834299873 & $-7,337199492$ & 1,827966457 & 67,29246805 & 0,989 \\
\hline 2010 & 64,306 & 1,808251496 & $-5,424754489$ & 1,846513182 & 70,22846596 & 5,922 \\
\hline 2011 & 68,735 & 1,837177937 & $-3,674355874$ & 1,865059907 & 73,29256266 & 4,558 \\
\hline 2012 & 81,698 & 1,912211425 & $-1,912211425$ & 1,883606632 & 76,49034716 & 5,208 \\
\hline 2013 & 86,623 & 1,937633221 & 1,937633221 & 1,920700082 & 83,31056542 & 3,312 \\
\hline 2014 & 90,762 & 1,957904057 & 3,915808115 & 1,939246807 & 86,94543947 & 3,817 \\
\hline 2015 & 96,114 & 1,982786652 & 5,948359955 & 1,957793532 & 90,73890457 & 5,375 \\
\hline 2016 & 93,496 & 1,970793031 & 7,883172124 & 1,976340257 & 94,69788009 & 1,202 \\
\hline 2017 & 94,005 & 1,973150954 & 9,865754769 & 1,994886982 & 98,82958734 & 4,825 \\
\hline 2018 & 101,127 & 2,004867124 & 12,02920274 & 2,013433707 & 103,1415627 & 2,015 \\
\hline TOTAL & 972,443 & 22,82584028 & 3,37550404 & & & 45,396 \\
\hline
\end{tabular}
exponential route $\omega_{t_{i}}=a b^{t_{i}}, a$ and $b$ will be [2]:

Table 10. The string of numbers regarding the Boeing's worldwide revenues, if this serial indicates an exponential route

$$
\begin{gathered}
\lg a=\frac{\sum_{i=1}^{n} \lg \omega_{i} \sum_{i=1}^{n} t_{i}^{2}-\sum_{i=1}^{n} t_{i} \lg \omega_{i} \sum_{i=1}^{n} t_{i}}{n \sum_{i=1}^{n} t_{i}{ }^{2}-\left(\sum_{i=1}^{n} t_{i}\right)^{2}}=\frac{22,82584028 \cdot 182-3,37550404 \cdot 0}{12 \cdot 182-0^{2}}=1,902153357 \\
\lg b=\frac{n \cdot \sum_{i=1}^{n} t_{i} \lg \omega_{i}-\sum_{i=1}^{n} \lg \omega_{i} \sum_{i=1}^{n} t_{i}}{n \sum_{i=1}^{n} t_{i}{ }^{2}-\left(\sum_{i=1}^{n} t_{i}\right)^{2}}=\frac{12 \cdot 3,37550404-22,82584028 \cdot 0}{12 \cdot 182-0^{2}}=0,018546725 \\
v_{\exp }=\left[\frac{\sum_{i=1}^{n}\left|\omega_{i}-\omega_{t_{i}}^{\exp }\right| \sum_{i=1}^{n} \omega_{i}}{n}\right] \cdot 100=\frac{\sum_{i=1}^{n}\left|\omega_{i}-\omega_{t_{i}}^{\exp }\right|}{\sum_{i=1}^{n} \omega_{i}} \cdot 100=\frac{45,396}{972,443} \cdot 100=4,67 \% \\
v_{I I I}=3,54 \%<v_{I I}=4,23 \%<v_{I}=4,25 \%<v_{\text {exp }}=4,67 \%
\end{gathered}
$$


The "mix" of the processing which unveils the evolution of the values concerning the Boeing's worldwide revenues, pursues a parabolic route of three degree $\omega_{t_{i}}=a+b \cdot t_{i}+c t_{i}^{2}+d t_{i}^{3}$

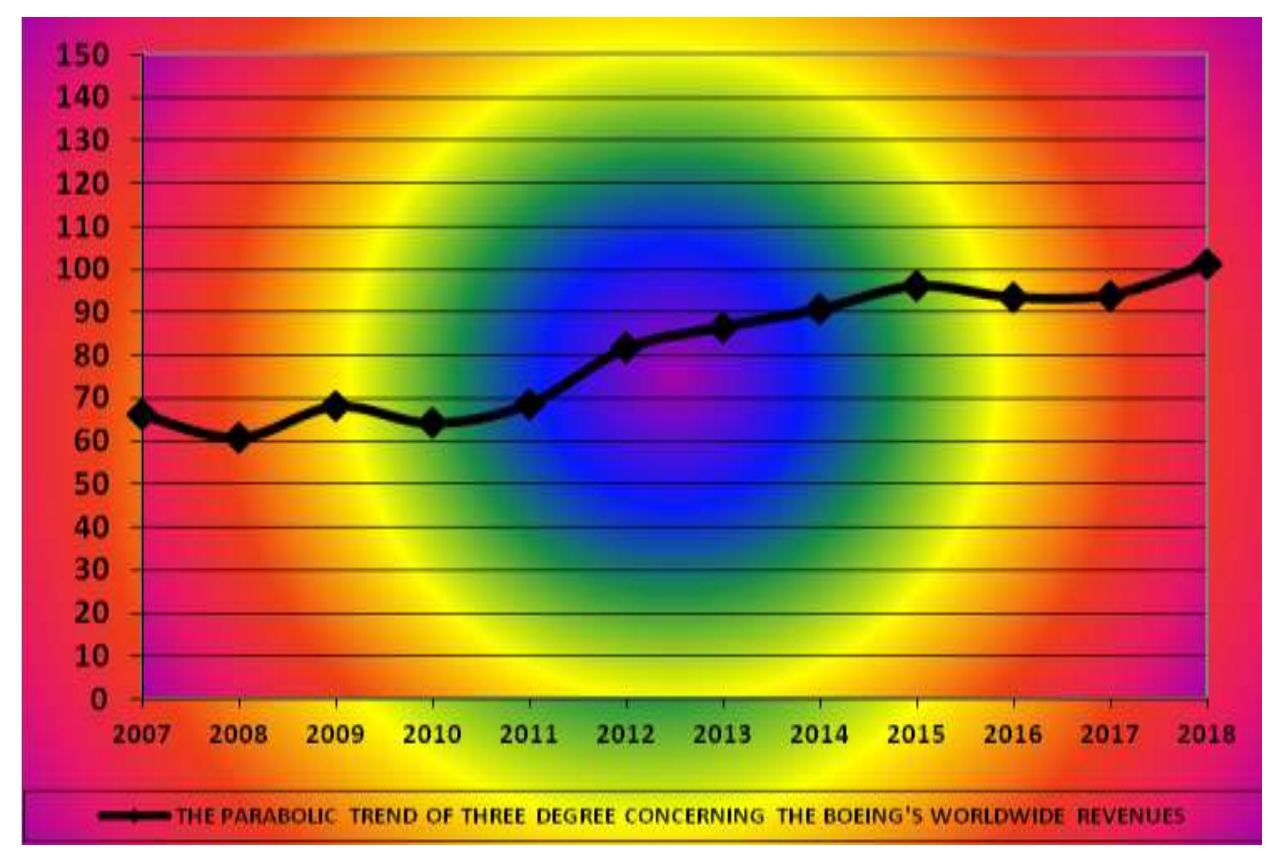
Graph 2 . The quadratic route of three degree for the packet unveiled by
the values which specify the evolution of the Boeing's worldwide revenues

\section{Conclusions}

The Boeing Company represents a „supersonic land” which incorporates a lot of qualities: gift, innovation, efficiency and performance. Because the management is applied to the highest quotas, the Boeing Company cans be a true model in the aeronautic and aerospace industry. We observe that the production will rise with 18 aircrafts in 2019, comparative to 2018, because the predicted stage in 2019 constitutes the level of 824 aircrafts. Also, the Boeing's Company worldwide revenues pursue a quadratic "itinerary" of three degree, in the spell of time 2007-2018. As novelty, the Boeing team specialized in the modern aerospace engineering inserted the new propulsion techniques: the electric propulsion, the ion propulsion and the computational fluid dynamics.

\section{References}

1. Baggins S. - "Modern Aerospace Engineering”, Willford Press Publishing House, Sydney, 2019.

2. Gauss C.F. - „Disquisitiones Arithmeticae and other papers on number theory”, english translation Springer Publishing House, New York, 1986.

3. Lombardi M. - „Strategic Airpower: the History of Bombers”, Boeing Press Publishing House, Chicago, 2014.

4. Mikel R. - "Aerospace and Aeronautical Engineering", Willford Press Publishing House, Sydney, 2019.

5. Sheng R. - "Systems Engineering for Aerospace - A Practical Approach", Academic Press, Elsevier Publishing House, Cambridge Massachusetts, 2019.

6. Yenne B. - "The Story of the Boeing Company, Updated Edition", Zenith Press Publishing House, Minneapolis, 2010. 